\title{
DISCRIMINATING GROUPS: A COMPREHENSIVE OVERVIEW
}

\author{
BENJAMIN FINE, ANTHONY M. GAGLIONE, ALEXEI MYASNIKOV AND \\ DENNIS SPELLMAN
}

\begin{abstract}
Discriminating groups were introduced by G.Baumslag, A.Myasnikov and V.Remeslennikov as an outgrowth of their theory of algebraic geometry over groups. However they have taken on a life of their own and have been an object of a considerable amount of study. In this paper we survey the large array results concerning the class of discriminating groups that have been developed over the past decade.
\end{abstract}

\section{Contents}

1. Discrimination and Discriminating Groups 1

2. Positive Examples 5

3. Negative Examples 8

4. Nontrivially Discriminating Groups 10

5. Squarelike Groups 13

6. Axiomatics and the Axiomatic Closure Property 16

$\begin{array}{ll}\text { 7. Varietal Discrimination } & 17\end{array}$

\section{Discrimination and Discriminating Groups}

If $G$ and $H$ are groups then $G$ separates $H$ provided that to every nontrivial element $h$ of $H$ there is a homomorphism $\varphi_{h}: H \rightarrow G$ such that $\varphi_{h}(h) \neq 1$. $G$ discriminates $H$ if to every finite nonempty set $S$ of nontrivial elements of $H$ there is a homomorphism $\varphi_{S}: H \rightarrow G$ such that

1991 Mathematics Subject Classification. Primary 20A05; Secondary 08C10, 20 E26. Key words and phrases. Discriminating group, squarelike group.

The research of the second author was partially supported by the Naval Academy Research Council.

This paper was prepared while two of the authors (B.Fine and A.Mysanikov) were visitors at the CRM in Barcelona. We'd like to thank the CRM for its hospitality. 
$\varphi_{S}(s) \neq 1$ for all $s \in S$. The group $\mathrm{G}$ is discriminating provided that it discriminates every group it separates.

Discriminating groups were invented by Baumslag, Myasnikov and Remeslennikov in [BMR 2] as a spin-off of their theory of algebraic geometry over groups [BMR 1]. Algebraic geometry over groups was created as a tool to attack the celebrated Tarski conjectures on the elementary theory of free groups. By analogy with classical algebraic geometry we may view the discrimination of $H$ by $G$ as an approximation to $H$ much like the localization of a ring at a prime. (Think of a set of generators for $H$ as a set of variables.)

The following is the main criterion for determining whether a group is discriminating.

Lemma 1.1. ([BMR 2]) $A$ group $G$ is discriminating if and only if $G$ discriminates $G \times G$.

Since this is fundamental we will give the proof which is very easy:

Since $G$ clearly separates $G \times G$ if it is discriminating it will discriminate $G \times G$.

Conversely suppose $G$ discriminates $G \times G$. By induction then $G$ will discriminate $G^{n}$. Suppose that $G$ separates a group $H$; we want to show that it discriminates $H$. Let $h_{1}, \ldots, h_{n}$ be nontrivial elements in $H$. Then for each there is a homomorphism $\phi_{i}: H \rightarrow G$ such that $\phi_{i}\left(h_{i}\right) \neq 1$. Taking $\phi=\phi_{1} \times \ldots \times \phi_{n}$ and using the assumption that $G$ discriminates $G^{n}$ gives us that $G$ discriminates $H$.

It is clear from the Lemma that if $G \times G$ embeds in $G$, then $G$ is discriminating. If this is the case we say that $G$ is trivially discriminating which we abbreviate as TD. In [FGMS 1] the question was asked if there are finitely generated non-trivially discriminating groups. We will give some examples of these later in the survey.

1.1. Some Ties to Logic. We now present some ties between discriminating groups and logic.

A universal sentence is one of the form $\forall \mathbf{x} \varphi(\mathbf{x})$ where $\mathbf{x}$ is a tuple of distinct variables and $\varphi(\mathbf{x})$ is a quantifier-free formula containing at most the variables in $\mathbf{x}$. Similarly, an existential sentence of $L$ is one of the form $\exists \mathbf{x} \varphi(\mathbf{x})$ where $\mathbf{x}$ and $\varphi(\mathbf{x})$ are as before. Vacuous quantifications are permitted and a quantifier-free sentence of $L$ is considered a special case both of universal sentences and existential sentences. Observe that the negation of a universal sentence is logically equivalent to an existential sentence and vice-versa. If $G$ is a group, then the universal theory of $G$, written $\mathrm{Th}_{\forall}(G)$, is the set of all universal sentences of $L$ true in $G$. Two groups $G$ and $H$ are universally equivalent or have the same universal theory, 
written $G \equiv_{\forall} H$, provided $\operatorname{Th}_{\forall}(G)=\mathrm{Th}_{\forall}(H)$. Observe that two groups satisfy precisely the same universal sentences if and only if they satisfy precisely the same existential sentences. Notice that it is easy to prove that all finitely generated free groups have the same universal theory.

Theorem 1.1. ([FGMS 1]): If $G$ is discriminating, then $G \times G \equiv{ }_{\forall} G$.

In order to better capture the axiomatic properties of discriminating groups we define.

Definition 1.1. ([FGMS 2]): The group $G$ is squarelike provided $G \times G$ $\equiv \forall$.

Hence, every discriminating group is squarelike (but, as we shall see, not conversely).

1.1 Some Ties to the Algebraic Geometry of Groups. As mentioned discriminating groups arose out of the study of the algebraic geometry over gorups. Here we present some of the ties.

A first order expression

$$
\left.\forall \mathbf{x} \wedge_{i}\left(w_{i}(\mathbf{x})=1\right) \rightarrow(u(\mathbf{x})=\mathbf{1})\right)
$$

where $u((\mathbf{x}))$ and the $w_{i}(\mathbf{x})$ are words on the variables and their formal inverses is called a quasi-identity

Observe that every identity $\forall \mathbf{x}(A(\mathbf{x})=\mathbf{a}(\mathbf{x}))$ is equivalent to a quasiidentity $\forall \mathbf{x}((1=1) \rightarrow(A(\mathbf{x})=a(\mathbf{x})))$. So laws and the group axioms themselves may be viewed as instances of quasi-identities.

Definition 1.2. The model class of a set of quasi-identities including the group axioms is a quasivariety of groups. If $G$ is a group the quaivariety generated by $G$ is denoted $\mathbf{q v a r}(\mathbf{G})$

Quasivarieties are examples of axiomatic classes. That is, they are model classes of sets of sentences of $L$, the language of group theory. Quasivarieties are closed under (unrestricted) direct products. They are also closed under subgroups since they have a set of universal axioms. Put another way, quasivarieties are axiomatic prevarieties. The class of all groups is a quasivariety of groups and the intersection of any family of quasivarieties is again a quasivariety. For that matter, the intersection of any family of universally axiomatizable model classes (i.e. axiomatic classes having a set of universal axioms) is again a universally axiomatizable model class. It follows that if $\mathcal{X}$ is any class of groups there is a least quasivariety, denoted qvar $(\mathcal{X})$, containing $\mathcal{X}$ and a least universally axiomatizable model class, denoted $\operatorname{ucl}(\mathcal{X})$, containing $\mathcal{X}$. We call qvar $(\mathcal{X})$ the quasivariety generated by $\mathcal{X}$ and we call $\operatorname{ucl}(\mathcal{X})$ the universal closure of $\mathcal{X}$. If $\mathcal{X}=\{G\}$ is a singleton 
we write $\operatorname{qvar}(G)$ and ucl $(G)$ for $\operatorname{qvar}(\mathcal{X})$ and $\operatorname{ucl}(\mathcal{X})$ respectively. qvar $(G)$ is the model class of the quasi-identities satisfied by $G$ and $\operatorname{ucl}(G)$ is the model class of the universal sentences satisfied by $G$. In general ucl $(G)$ is a proper subclass of $q \operatorname{var}(G)$. (The more properties a structure is required to satisfy the fewer structures can satisfy them in general.)

One final concept and then the result.

Definition 1.4. The group $G$ is equationally Noetherian provided for every nastural number $n \in N$ and every system $S=1$ of equations in $n$ unknowns there is a finite subset $S_{0} \subseteq S$ such that $V_{G}(S)=V_{G}\left(S_{0}\right)$.

Examples of equationally Noetherian groups are groups linear over a commutative Noetherian ring with 1. In particular, every group linear over a field is equationally Noetherian.

Tying all of these together is:

Theorem 1.3 (FGMS 1). Let $G$ be a finitely generated equationally Noetherian group. Then $G$ is discriminating if and only if qvar $(G)=u c l(G)$.

1.3 Abelian Discriminating Groups. In the original paper by Baumslag,Myasnikov and Remeslennikov [BMR 2] the question was raised as to whether the abelian discriminating groups can be characterized. In [FGMS 1] this was extended to ask whether the abelian squarelike groups could be characterized. If there is any torsion this can be answered imeediately.

Lemma 1.3.1. Suppose the set of nontrivial elements of finite order in the group $G$ is finite and nonempty. Then $G$ is not squarelike. Hence, $G$ is not discriminating.

We immediately deduce that no nontrivial finitely generated nilpotent group with torsion can be squarelike. In particular, no nontrivial finitely generated abelian group with torsion and no nontrivial finite group can be squarelike. In the torsion-free case we always get discrimination.

Lemma 1.3.2. Every torsion free abelian group is discriminating. Hence, every torsion free abelian group is squarelike.

So, among finitely generated abelian groups, the discriminating groups and the squarelike groups are precisely the torsion-free ones. A moment's reflection produces nontrivial discriminating abelian groups ( necessarily not finitely generated) with torsion. For example, every group free of infinite rank in the variety of abelian groups of exponent dividing $n$ for any fixed integer $n>1$ is trivially discriminating.

Baumslag, Myasnikov and Remeslennikov in [BMR 2] give a partial answer to the total characterization of abelian discriminaitng groups. We must introduce the Szmielew invariants of an abelian group. Given an 
integer $m>0$ and a family of elements $\left(a_{i}\right)$ in an additively written abelian group $A,\left(a_{i}\right)$ is linearly independent modulo $\mathbf{m}$ provided $\Sigma_{i} n_{i} a_{i}=0$ implies $n_{i} \equiv 0(\bmod m)$ for all $i ;\left(a_{i}\right)$ is linearly independent modulo $\mathbf{m}$ in the stronger sense provided $\Sigma_{i} n_{i} a_{i} \in m A$ implies the coefficients $n_{i} \equiv 0(\bmod m)$ for all $i$. For each prime $p$ and positive integer $k$ we define three ranks each of which is a nonnegative integer or the symbol $\infty$.

(1) $\rho^{(1)}[p, k](A)$ is the maximum number of elements of $A$ of order $p^{k}$ and linearly independent modulo $p^{k}$

(2) $\rho^{(2)}[p, k](A)$ is the maximum number of elements of $A$ linearly independent modulo $p^{k}$ in the stronger sense.

(3) $\rho^{(3)}[p, k](A)$ is the maximum number of elements of $A$ of order $p^{k}$ and linearly independent modulo $p^{k}$ in the stronger sense.

Proposition 1.3.1. (Szmielew [S]) Let $A$ and $B$ be abelian groups. Then $A$ and $B$ are elementarily equivalent if and only if the following two properties are satisfied.

(1) $A$ and $B$ either both have finite exponent or both have infinite exponent.

(2) For all primes $p$ and positive integers $\left.k, \rho^{(i)}[p, k](A)=\rho^{(i)}[p, k](B)\right)$ for $i=1,2,3$.

Proposition 1.3.2. ([BMR 2]) Let $A$ be a torsion abelian group such that, for each prime $p$, the p-primary component of $A$ modulo its maximal divisible subgroup contains no nontrivial elements of infinite p-height. Then $A$ is discriminating if and only if, for each prime $p$, the following two properties are satisfied.

(1) For all positive integers $k, \rho^{(1)}[p, k](A)$ is either 0 or $\infty$.

(2) The rank of the maximal divisible subgroup of the p-primary component of $A$ is either 0 or infinite.

Extending the ideas of the [BMR 2] paper, Fine, Gaglione,Mayasnikov and Spellman [FGMS 2] developed a complete characterization of abelian squarelike groups.

Proposition 1.3.3. ([FGMS 2]) Let $A$ be an abelian group. Then $A$ is squarelike if and only if, for each prime $p$ and positive integer $k, \rho^{(1)}[p, k](A)$ is either 0 or $\infty$.

\section{Positive Examples of Discrimninating Groups}

In the theory as applied to the algebraic geometry of groups it is important to know both positive examples of discriminating groups and negative examples. The original positive examples of discriminating groups were either abelian or trivially discriminating. Recall that a group is trivially 
discriminating if it embeds its direct square. In this section we present these positive examples. We will return later to examples of nontrivailly discriminating groups.

Most of the early discovered postive examples were also "universal type groups"

(1) EXAMPLE 1: Abelian Groups:

Torsion-free abelian groups and the torsion abelian groups described above. ([BMR 2]. )

(2) EXAMPLE 2: Higman's universal group $\mathcal{H}$.

Higman constructed this group $\mathcal{H}[\mathrm{Hi}]$ which is a finitely presented group which embeds every other finitely presented group.

Since this group is finitely presented its direct square is also finitely presented. Therefore it embeds its direct square and hence is trivially discriminating.

(3) EXAMPLE 3: Thompson's Group $F$.

This group consists of all orientation preserving piecewise linear homeomorphisms from the unit interval $[0,1]$ onto itself that are differentiable except at finitely many dyadic rational numbers and such that on intervals of differentiability the derivatives are powers of 2 is finitely presented. As in Higman's group, this group embeds its direct square and hence is trivially discriminating. This is also finitely presented.

(4) EXAMPLE 4: The commutator subgroup of the Gupta-Sidki-Grigorchuk Group.

For each odd prime $p$, Gupta and Sidki and independently Grigorchuk constructed a group $H_{p}$ which is a subgroup of the group of automorphisms of a rooted tree. $H_{p}$ is a 2-generator infinite $p$-group. Consider the commutator subgroup $H_{p}^{\prime}$ of $H_{p}$. It can be shown that $H_{p}^{\prime}$, while finitely generated, is not finitely presentable. It can be shown that $H_{p}^{\prime}$ is trivially discriminating.

(5) EXAMPLE 5: Groups which are isomorphic to their direct squares.

Examples of nontrivial finitely generated groups isomorphic to their direct squares were first constructed by Tyrer Jones [J] and subsequently by Hirshon and Meier [HM]. The question of whether or not there exists a finitely presented group isomorphic to its direct square remains open.

(6) EXAMPLE 6: The Grigorchuk group of intermediate growth. Grigorchuk developed a group of intermediate growth (see [FGMS 1]). It was 
shown in that paper, due to conversations with Grigorchuk, that this group is discriminating. Whether is is trivially discriminating or not is open.

(7) EXAMPLE 7: It was shown in [BFGS 1] that

Lemma 2.1. A simple discriminating group is trivially discriminating.

Then: For each integer $n \geq 2$ and each integer $r \geq 1$, Higman defined a group $G_{n, r}$ (see [Hi 2]) as follows. Let $\mathcal{V}_{n}$ be the variety of all algebras with one $n$-ary operation $\lambda$ and $n$ unary operations $\alpha_{1}, \ldots, \alpha_{n}$ subject to the laws $\lambda\left(\alpha_{1}(x), \ldots, \alpha_{n}(x)\right)=x$ and $\alpha_{i}\left(\lambda\left(x_{1}, \ldots, x_{n}\right)\right)=x_{i}, i=1, \ldots, n$. If $V_{n, r}$ is an algebra free on $\mathrm{r}$ generators in $\mathcal{V}_{n}$, then we let $G_{n, r}=\operatorname{Aut}\left(V_{n, r}\right)$ be its group of automorphisms. Higman proved that the $G_{n, r}$ are finitely presented, when $n$ is even $G_{n, r}$ is simple and when $n$ is odd $G_{n, r}$ contains a simple subgroup $G_{n, r}^{+}$of index 2 . Setting $G_{n, r}^{+}=G_{n, r}$ when $\mathrm{n}$ is even, he showed that, for fixed $r, G_{m, r}^{+} \cong G_{n, r}^{+}$implies $m=n$. Thus he found an infinite family of finitely presented infinite simple groups. The $G_{n, r}$ are all trivially discriminating.

(8) EXAMPLE 8: The group $S_{\omega}$ of all permutations of $\mathbb{N}$ which move only finitely many integers and the subgroup $A_{\omega}$ of even permutations are each trivially discriminating

(9) EXAMPLE 9: The existence of a finitely generated but not finitely presentable trivially discriminating group $G_{1}$ (e.g. the commutator subgroup of a Gupta-Sidki-Grigorchuk group) together with the existence of a universal finitely presented group (Higman's Group $G_{2}$ ) allowed us to construct in [FGS 4] a group $G=G_{1} \times G_{2}$ which is proven in that paper to be a finitely generated discriminating group which is neither finitely presented nor equationally Noetherian.

This led to the obvious question:

DOES THERE EXIST A FINITELY PRESENTED NONTRIVIALLY DISCRIMINATING GROUP?

We will return to this shortly. 


\section{Negative Examples: Examples of Nondiscriminating Groups}

In the application of algebraic geometry it is as important to know both whther a group is discriminating and whether it is not discriminating. This further raised general questions on nondiscrimination of various classes of groups in particular groups in various varieties.

The general idea to show nondiscrimination of a group is to show some universal property which is true in $G$ but cannot be true in $G \times G$ or to find a number - dimension etc. - which is additive so cannot hold in $G \times G$ and in $G$.

Definition 3.1. The group $G$ is commutative transitive or $C T$ provided the centralizer of every nontrivial element is abelian.

We observe that the group $G$ is $\mathrm{CT}$ if and only if it satisfies the universal sentence

$$
\forall x, y, z(((y \neq 1) \wedge(x y=y x) \wedge(y z=z y)) \rightarrow(x z=z x)) .
$$

If $G$ is non-abelian then $G \times G$ cannot satisfy this sentence. Therefore the following lemma follows directly.

Lemma 3.1. A nonabelian CT group is nondiscriminating.

In particular we obtain the negative examples.

(1) Any torsion-free hyperbolic group and in particular any nonabelian free group is nondiscriminating

(2) Nonabelian free solvable groups and their nonabelian subgroups are nondiscriminating.

(3) The free product of two nondiscriminating groups is nondiscriminating.

In another direction, if we think of discrimination as having the direct square $G \times G$ almost embedding in the group $G$, then this would be impossible for a finite group. In particular it is impossible if the torsion elements form a finite subgroup.

Lemma 3.2. If the torsion elements of a group $G$ form a finite subgroup then $G$ is nondiscriminating. 
This leads to the further negative examples.

(4) Any finitely generated nilpotent group with nontrivial torsion is nondiscriminating

(5) Any finite group is nondiscriminating.

One of the major questions raised in the early work on discriminating groups ([BMR 2], [FGMS ]) was whether of not a nonabelian nilpotent group can be discriminating. The full result was:

Theorem 3.1. A finitely generated nilpotent group is discriminating if and only if it is torsion-free abelian.

There were several stages to obtaining the total final result (Theorem 3.1) although the final proof was rather direct. In [FGMS 1] it was shown that nonabelian finitley generated free nilpotent groups are nondiscriminating. To do this an extension of commutative transitivity was introduced. Myasnikov and Shumyatsky ([MS]) introduced the concept of centralizer dimensions (see $[\mathrm{MS}]$ ) and showed that a group with finite centralizer dimension is nondiscriminating. As a consequence of this if follows that a finitely generated nilpotent group is discriminating if and only if it is torsion-free abelian. Their method yileded the following further result.

Theorem 3.2. A finitely generated linear group is discriminating only if it is torsion-free abelian.

Independently Baumslag,Fine,Gaglione and Spellman using vector space dimension proved that a nilpotent group is discriminating if and only if its Malcev completion is discriminating. Theorem 3.1 can then be recovered from this result.

Theorem 3.3. ([BFGS 2]) The Malcev completion of a finitely generated torsion-free nilpotent group is discriminating if and only if it is abelian.

The nondiscrimination of nonabelian nilpotent groups leads to the question as to whether any nonabelian group in a variety must be nondiscriminating. There is some further evidence for this. Kassabov $[\mathrm{K}]$ proved it for solvable groups.

Theorem 3.4. ([K]) A finitely generated solvable group is discriminating only if it is torsion-free abelian.

Further in this direction, Baumslag, Fine, Gaglione and Spellman [BFGS 1]n proved some general results on nondiscrimination of relatively free groups. 
Theorem 3.5 (BFGS 1). Let $F$ be a non-abelian free group and let $R$ be a non-trivial, normal subgroup of $F$ such that $F / R$ is torsion-free and contains a free abelian group of rank two. Then $F / V(R)$ is not discriminating.

Theorem 3.6 (BFGS 1). Let $\mathcal{V}$ and $\mathcal{U}$ be two varieties. If the variety $\mathcal{V}$ contains the infinite cyclic group, then the non-abelian free groups in the product variety $\mathcal{U V}$ are not discriminating.

Theorem 3.7 (BFGS 1). For all sufficiently large primes $p$, the non-abelian free groups $G$ in the variety of all groups of exponent $p$ are not discriminating.

We pose the following question:

\section{DOES THERE EXIST A NONABELIAN, DISCRIMINATING, RELA-} TIVELY FREE GROUP?

\section{Nontrivially Discriminating Groups}

Recall the quesion of whether there exists a finitely generated nontrivially discriminating group. That is a finitely generated discriminating group which does not embed its direct square. One possibility (still open) was the Grigorchuk groups of intermediate growth. Here we give other examples done with Peter Neumann and Gilbert Baumslag. We present two classes of examples. The groups in the first class are finitely presented. The second is a class of groups studied by B.H. Neumann which are non-trivally discriminating and finitely generated but probably not finitely presented.

EXAMPLE 1: To describe the first class of groups let $X$ be a nonabelian, finitely generated, torsion free, nilpotent group and let $Y$ be one of the infinite simple groups, $G_{n, r}$ as described by Higman. The following properties of $X$ and $Y$ are needed:

(a) $X$ and $Y$ are finitely presented;

(b) $X$ is residually finite;

(c) every finite group is embeddable in $Y$;

(d) $X$ is not embeddable in $Y$; in fact, a torsion free nilpotent subgroup of $Y$ is abelian;

(e) $Y \times Y \times Y$ is embeddable in $Y$. (This follows from the fact that $\mathcal{V}_{n, r} \cong \mathcal{V}_{n, s}$ in the notation of [GH2] if and only if $r \equiv s \bmod (n-1)$.)

Our class $\mathcal{G}_{1}$ consists of the groups $X \times Y$.

Theorem 4.1 (FGS 4). Each group $G \in \mathcal{G}_{1}$ is a nonabelian, finitely presented non-trivially discriminating group.

We present the proof to exhibite the techniques involved. 
Proof. Clearly each group $G \in \mathcal{G}_{1}$ is non-abelian and finitely presented. Then suppose $G \in \mathcal{G}_{1}$. We must show show that $G$ is discriminating but that $G$ does not embed its direct square.

Since $G=X \times Y$ with $X$ and $Y$ as described above to prove that $G$ is discriminating it suffices to show that $Y$ discriminates any group of the form $W \times Y \times Y$ where $W$ is residually finite. This is sufficient for then $G=X \times Y$ will discriminate its direct square and hence be discriminating. For this purpose, let $h_{1}, \ldots, h_{n}$ be finitely many non-identity elements of $W \times Y \times Y$ Write $h_{i}=\left(a_{i}, b_{i}\right)$ where $a_{i} \in W$ and $b_{i} \in Y \times Y$. Without loss of generality, we may suppose that $a_{i} \neq 1$ for $1 \leq i \leq m$ and $a_{i}=1$ for $m+1 \leq i \leq n$. Since $W$ is residually finite, there exists a finite group $V$ and a homomorphism $\alpha: W \rightarrow V$ such that $\alpha\left(a_{i}\right) \neq 1$ for $1 \leq i \leq m$. By (c) above we may embed $V$ into $Y$ and so we get a homomorphism $\beta: W \rightarrow Y$ such that $\beta\left(a_{i}\right) \neq 1$ for $1 \leq i \leq m$. Let $\gamma$ be the homomorphism $\gamma: W \times Y \times Y \rightarrow Y \times Y \times Y$ defined by $(w, y, y)) \stackrel{\gamma}{\rightarrow}(\beta(w), y, y)$. By (e) above let $\delta$ be an embedding of $Y \times Y \times Y$ into $Y$. If we put $\varphi=\delta \circ \gamma$, then clearly $\varphi$ is a homomorphism such that $\varphi\left(h_{i}\right) \neq 1$ for all $i=1, \ldots, n$. Thus $Y$ discriminates $W \times Y \times Y$ and therefore it follows that $G$ discriminates $G \times G$. Thus $G$ is discriminating.

To show that is nontrivially discriminating we must show that $G \times G$ is not embeddable into $G$. Assume to deduce a contradiction that $G \times G \hookrightarrow G$. Restricting the embedding we get an embedding $X \times X \hookrightarrow X \times Y$. Letting $A$ be the projection of $X \times X$ into $Y$, then $X \times X \leq X \times A$, so $X^{\prime} \times X^{\prime} \leq X^{\prime} \times A^{\prime}$. But this tells us that if $h=$ the Hirsch length of the commutator subgroup $X^{\prime}$, then $h \leq 2 h \leq h$ since $A^{\prime}$ is finite. This contradicts the fact that $X$ is non-abelian and torsion free nilpotent. Therefore $G \times G$ is not embeddable into $G$ and hence is nontrivially discriminating.

EXAMPLE 2: The second class of non-trivally discriminating groups, which we will denote $\mathcal{G}_{2}$ was introduced by B.Neumann in a different context. Let $n_{1}, n_{2}, \ldots$ be a strictly increasing sequence of odd positive integers with $n_{1} \geq 5$. Let $\Lambda_{r}=\left\{1,2, \ldots, n_{r}\right\}$ and let $G_{r}$ be the alternating group on $\Lambda_{r}$. In $G_{r}$ consider the two elements

$$
x_{r}=(123), \quad y_{r}=\left(12 \ldots n_{r}\right) .
$$

In the unrestricted direct product $\prod_{r} G_{r}$ define

$$
x=\left(x_{1}, x_{2}, \ldots\right) \quad, y=\left(y_{1}, y_{2}, \ldots\right)
$$

and let

$$
G=<x, y>\subseteq \prod_{r} G_{r} .
$$


B.H. Neumann proved that the restricted direct product $D=G_{1} \times G_{2} \times$ ... is a subgroup of $G$. The class $\mathcal{G}_{2}$ consists of all the groups $G$ constructed as above.

Theorem 4.2. Each group $G \in \mathcal{G}_{2}$ is a finitely generated non-trivially discriminating group.

The methods used in constructing these non-TD examples can be generalized via the use of ascending chains of subgroups.

Theorem 4.3 (BFGS 1). Let $U$ be the union of a properly ascending chain of subgroups

$$
U_{1}<U_{2}<\cdots<U_{n}<\ldots
$$

and let $P$ be the unrestricted direct product of the $U_{i}$ :

$$
P=\bar{\prod}_{i=1}^{\infty} U_{i}
$$

Furthermore let $Q$ be the restricted direct product

$$
Q=\prod_{i=1}^{\infty} U_{i}
$$

Then every subgroup $G$ of $P$ containing $Q$ is discriminating (where here we view $Q$ as a subgroup of $P$ ).

Proof. The proof of this result is straightforward. To this end, let $D=G \times G$ and let $d_{1}=\left(a_{1}, b_{1}\right), \ldots, d_{k}=\left(a_{k}, b_{k}\right)$ be finitely many non-trivial elements of $D$. Each of the elements of $G$ can be viewed as a sequence of elements whose $n-t h$ term is contained in $U_{n}$. Since $G$ contains $Q$ and the the series of subgroups $U_{j}$ is increasing, there exists an integer $\alpha$ such that all of $a_{1}, b_{1}, \ldots, a_{k}, b_{k} \in U_{\alpha}$. We define now a homomorphism of $D$ into $G$ by projecting the first coordinates of the elements of $D$ to $U_{\alpha}$ and the second coordinates to $U_{\alpha+1}$. The upshot of this is that we have defined a homomorphism $\theta$ of $G \times G$ into $U_{\alpha} \times U_{\alpha+1}$. If we view $U_{\alpha} \times U_{\alpha+1}$ as a subgroup of $G$, it follows that $\theta$ is a homomorphism of $G \times G$ into $G$ mapping the given elements $d_{1}, \ldots, d_{k}$ non-trivially. This completes the proof.

This final theorem can be used to recover the proofs of the preceding examples and to obtain the fact that there exists continuously many 2generator non-TD discriminating groups. In particular let $A_{i}$ denote the alternating group of degree $i$ and let $G_{r}$ be the subgroup defined in the proof of Theorem 4.2. Then $G_{s}$ contains the restricted direct product of the $A_{n_{i}}$ and that

$$
G_{s} \cong G_{t} \text { if and only if } s=t
$$


This implies that there are continuously many non-isomorphic 2-generator groups. Theorem 4.3 then applies to these groups. Further B.H. Neumann proved that the only finite normal subgroups of $G_{r}$ are direct products of finitely many of the $A_{n_{i}}$. This implies, remembering that the alternating groups involved here are all simple, that none of the $G_{r}$ are TD. We remark here that it has recently been proved that none of the groups $G_{s}$ is finitely presented ( G.Baumslag and C.F. Miller).

Corollary 4.1. The groups $G_{r}$ are discriminating and further are not TD. Thus there exist continuously many 2-generator nontrivially discriminating groups.

\section{Squarelike Groups}

In order to better capture the axiomatic properties of discriminating groups the class of squarelike groups were introduced. These are groups which share the same universal theory as their direct squares.

Definition 5.1. ([FGMS 2]): The group $G$ is squarelike provided $G \times G$ $\equiv_{\forall} G$.

Hence, every discriminating group is squarelike (but, as we shall see), not conversely).

Theorem 5.1. The class of discriminating groups is a proper subclass of the class of squarelike groups.

To prove this we explicitly construct a discriminating group which is provably non-squarelike. This can also be proved with an axiomatic argument done below.

EXAMPLE: Let $H$ be the subgroup of the group of all permutations of the set $\mathbb{Z}$ of integers generated by the 3 -cycle $\xi=(012)$ and the translation $\eta(n)=n+1$ for all $n \in \mathbb{Z} . H=\langle\xi, \eta\rangle$ can also be described as the semidirect product of the group $M$, of all even parity permutations within the group $N$ of all permutations of the set $\mathbb{Z}$ of integers which move only finitely many integers, by an infinite cyclic group $C=<c$; $>$ where the automorphism $\alpha(c): M \rightarrow M$ acts by $\alpha(c)(\pi)(n)=\pi(n-1)+1$. (We say that $\alpha(\mathrm{c})$ acts by translation by 1.) Note that any bijection between $\mathbb{N}$ and $\mathbb{Z}$ induces an isomorphism between the infinite alternating group $A_{\omega}$ and $M$. $H$ first appeared in print in the same paper of B.H. Neumann in which the uncountably many nontrivially discriminating groups $G_{\mathbf{n}}$ exhibited before were introduced. B.H. Neumann observed that (independent of $\mathbf{n}$ ) if $K_{0}$ is the restricted direct product of the family $\left(A_{n(r)}\right)_{r \in \mathbb{N}}$ of alternating groups, 
then the quotient of $G=G_{\mathbf{n}}$ modulo $K_{0}$ is isomorphic to $H$. It can be proved that $\mathrm{H}$ is nondiscriminating however $\mathrm{H}$ is squarelike.

In an earlier paper this was done differently. Recall that a class of groups is axiomatic if it is the model class of a set of first order sentences (axioms). It can be shown that a class of groups is axiomatic if it is closed under ultraproducts and elementary equivalence.

Theorem 5.2. ([FGMS 2]) The class of squarelike groups is axiomatic but the class of discriminating groups is not axiomatic.

To prove that the discriminating groups are nonaxiomatic a specific example of a nondiscriminating group (however squarelike) was constructed which was elementarily equivalent to a discriminating group. Hence the discriminating groups are not closed under elementary equivalence.

Originally the fact that the squarelike groups are axiomatic was proved by using the closure properties noted above. However subsequently V.H.Dyson [D] discovered an explicit axiom schema for the class of squarelike groups. To each ordered pair $(\mathbf{w}, \mathbf{u})$ of finite tuples of words on a fixed but arbitrary finite set $\left\{x_{1}, \ldots, x_{n}\right\}$ of distinct variables and their formal inverses we assign the following sentence $\sigma(\mathbf{w}, \mathbf{u})$ of $L$.

$\forall \mathbf{x}\left(\bigwedge_{i}\left(w_{i}(\mathbf{x})=1\right) \rightarrow \bigvee_{j}\left(u_{j}(\mathbf{x})=1\right)\right) \rightarrow \bigvee_{j} \forall \mathbf{x}\left(\left(\bigwedge_{i} w_{i}(\mathbf{x})=1\right) \rightarrow\left(u_{j}(\mathbf{x})=1\right)\right)$

The contrapositive of $\sigma(\mathbf{w}, \mathbf{u})$ is (up to logical equivalence) the sentence $\tau(\mathbf{w}, \mathbf{u})$ asserting

$$
\left.\left.\bigwedge_{j} \exists \mathbf{x}\left(\bigwedge_{i} w_{i}(\mathbf{x})=1\right) \wedge\left(u_{j}(\mathbf{x}) \neq 1\right)\right) \rightarrow \exists \mathbf{x}\left(\bigwedge_{i} w_{i}(\mathbf{x})=1\right) \wedge \bigwedge_{i}\left(u_{j}(\mathbf{x}) \neq 1\right)\right) .
$$

Theorem 5.3. (Huber-Dyson [D]) The class of squarelike groups is the model class of the group axioms and the sentences $\sigma(\boldsymbol{w}, \boldsymbol{u})$. Hence, the class of squarelike groups is axiomatic.

Although the squarelike groups properly contain the discriminating groups they are very close and in fact correspond in the presence of finite presentation. In particular:

Theorem 5.4. ([FGMS 2]) Let $G$ be a finitely presented group. Then $G$ is discriminating if and only if $G$ is squarelike.

We give the proof because its very pretty

Proof. Suppose $G$ is a finitely presented group. If it is discriminating, it is squarelike. Now we suppose that $G$ is squarelike and we must show that it is discriminating. 
Let

$$
G=<x_{1}, \ldots, x_{n} ; R_{1}, \ldots, R_{m}>
$$

be a finite presentation for $G$ where $R_{i}=R_{i}\left(x_{1}, \ldots, x_{n}\right)$ are words in $x_{1}, . ., x_{n}$. To show that $G$ is discriminating we must show that $G$ discriminates $G \times G$.

A finite presentation for $G \times G$ is then given by

$$
\begin{gathered}
G \times G=<x_{1}, \ldots, x_{n}, y_{1}, \ldots, y_{n} ; R_{1}\left(x_{1}, \ldots x_{n}\right)=1, \ldots, R_{m}\left(x_{1}, . ., x_{n}\right)=1, \\
R_{1}\left(y_{1}, \ldots y_{n}\right)=1, \ldots, R_{m}\left(y_{1}, . ., y_{n}\right)=1,\left[x_{i}, y_{j}\right]=1, i, j=1, . ., n>.
\end{gathered}
$$

Now suppose $W_{1}, \ldots, W_{k}$ are non-trivial elements of $G \times G$. Then each $W_{i}, i=1, \ldots k$ is given by $W_{i}=W_{i}\left(x_{1}, \ldots, x_{n}, y_{1}, \ldots, y_{n}\right)$, a word in the given generators of $G \times G$. Consider now the existential sentence

$$
\begin{gathered}
\exists x_{1}, \ldots, x_{n}, y_{1}, . ., y_{n}\left(\left(\bigwedge_{i=1}^{m} R_{i}\left(x_{1}, . ., x_{n}\right)=1\right) \wedge\left(\bigwedge_{i=1}^{m} R_{i}\left(y_{1}, . ., y_{n}\right)=1\right)\right. \\
\left.\wedge\left(\bigwedge_{i, j}\left[x_{i}, y_{j}\right]=1\right) \wedge\left(\bigwedge_{i=1}^{k} W_{i}\left(x_{1}, \ldots, x_{n}, y_{1}, \ldots, y_{n}\right) \neq 1\right)\right)
\end{gathered}
$$

This existential sentence is clearly true in $G \times G$. Since $G$ is squarelike, $G$ and $G \times G$ have the same universal theory. Hence they have the same existential theory and therefore the above existential sentence is true in $G$. Therefore there exists elements $a_{1}, \ldots, a_{n}, b_{1}, \ldots, b_{n}$ in $G$ such that $R_{i}\left(a_{1}, \ldots, a_{n}\right)=1$ for $i=1, \ldots, m ; R_{i}\left(b_{1}, \ldots, b_{n}\right)=1$ for $i=1, \ldots, m ;\left[a_{i}, b_{j}\right]=1$ for $i, j=1, \ldots, n$ and $W_{i}\left(a_{1}, \ldots, a_{n}, b_{1}, \ldots, b_{n}\right) \neq 1$ for $i=1, \ldots, k$. The map from $G \times G$ to $G$ given by mapping $x_{i}$ to $a_{i}$ and $y_{i}$ to $b_{i}$ for $i=1, \ldots, n$ defines a homomorphism for which the images of $W_{1}, . ., W_{k}$ are non-trivial. Hence $G$ discriminates $G \times G$ and therefore $G$ is discriminating.

Finally recall that

Theorem (FGMS 1). Let $G$ be a finitely generated equationally Noetherian group. Then $G$ is discriminating if and only if $\operatorname{qvar}(G)=u \operatorname{cl}(G)$.

For squarelike groups we do not need the equationally noetherian condition.

Theorem 5.5. ([FGMS 2]) Let $G$ be a group. The following three conditions are pairwise equivalent.

- $G$ is squarelike.

- $u c l(G)=\operatorname{qvar}(G)$.

- There is a discriminating group $H$ such that $G \equiv_{\forall} H$.

Therefore the squarelike groups are the universal closure of the discriminating groups. In fact they are the axiomatic closure. 


\section{Axiomatics and the Axiomatic Closure Property}

The least axiomatic class containing the discriminating groups is the class of those groups $G$ for which there exists a discriminating group $H$ with $G \equiv H$. Here $\equiv$ denotes elementary equivalence. We call this class the axiomatic closure of $G$.

In [FGS 2] it was established that the squarelike groups are precisely the axiomatic closure of the discriminating groups.

Theorem 6.1. ([FGS 2]) Let $G$ be a group. Then $G$ is squarelike if and only if there is a discriminating group $H$ with $G \equiv H$. Hence the class of squarelike groups is the axiomatic closure of the class of discriminating groups.

Proof. We outline the proof which uses ultrapowers and ultralimits.

If $G \equiv H$, then $G \equiv_{\forall} H$; so, $G$ elementarily equivalent to a discriminating group implies $G$ is squarelike.

Assume now that $G_{0}$ is a squarelike group. Then $G_{0} \forall\left(G_{0} \times G_{0}\right)$ so there is an ultrapower $G_{1}=G_{0}^{I} / D$ admitting an embedding $\varphi_{1}: G_{0} \times G_{0} \rightarrow G_{1}$. Let $d_{0,1}: G_{0} \rightarrow G_{1}$ be the canonical embedding and let $G_{2}=G_{1}^{I} / D$. Then $\varphi_{1}$ induces $\varphi_{2}: G_{1} \times G_{1} \rightarrow G_{2}$ and a diagram chase convinces one that the square

$$
\begin{array}{ccccc} 
& & \multicolumn{1}{c}{\phi_{1}} & & \\
d_{0,1} \times d_{0,1} & \times G_{0} & \rightarrow & G_{1} & \\
& \downarrow & & \downarrow & d_{1,2} \\
& G_{1} \times G_{1} & \rightarrow & G_{2} & \\
& & & \phi_{2} &
\end{array}
$$

is commutative where $d_{1,2}: G_{1} \rightarrow G_{2}$ is the canonical embedding.

We may iterate a countable infinity of times. Taking $G_{\omega}$ to be the ultralimit of $G_{0}$ with respect to the constant sequence of ultrafilters $(D, D, \ldots, D, \ldots)$, we get an embedding $G_{\omega} \times G_{\omega} \rightarrow G_{\omega}$ so that $G_{\omega}$ is discriminating. But the limit map $d_{0, \omega}: G_{0} \rightarrow G_{\omega}$ is elementary. Hence, $G_{0} \equiv G_{\omega}$.

There is a great deal of further axiomatic information about both squarelike and discriminating groups (see [FGS 3]). As we have seen in the previous sections the class of squarelike groups is axiomatic, that is is given by a set of first -order axioms while the class of sqarelike groups is not. Originally this was proved in [FGMS 2] using Malcev's conditon for axiomability. Subsequently an explicit set of axioms was given by V.H. Dyson (see [FGS 3]). P. Schupp rasied the question of whether the theory of squarelike groups was finitely aximomatizable, that is the axiom set can be taken to be finite. This was shown to be negative ([FGS 3]). 
Theorem 6.2. ([FGS 3]) The theory of squarelike groups is not finitely axiomatizable.

Recall that if $\mathcal{X}$ is a class of groups then the first-order theory of $\mathcal{X}$ is decidable if there exists a recursive algorithm which, given a sentence $\phi$ of $L_{0}$ decides whether or not $\phi$ is true in every group in $\mathcal{X}$. As a consequence of the undecidability of the word problem (see [FGS 3]) it was shown that the theory of the squarelike groups is undecidable.

Theorem 6.3. ([FGS 3]) The theory of squarelike groups is undecidable.

\section{VARIETAL DiscRimination}

There is an older notion of discrimination, which we call varietal discrimination, that was also introduced by G. Baumslag jointly with Bernard, Hanna and Peter Neumann (see [N]). The properties of this type of discrimination are described in the book of Hanna Neumann $[\mathrm{N}]$ and play a role in the structure of product varieties.

In [FGS 5] the relationship between these various notions of discrimination was examined. If we let $\mathcal{D}$ denote the class of discriminating groups, $\mathcal{S}$ the class of squarelike groups and $\mathcal{V D}$ the class of varietally discriminating groups the main result is that

$$
\mathcal{D} \subset \mathcal{S} \subset \mathcal{V D}
$$

and all inclusions are proper.

In order to prove that $\mathcal{S} \subset \mathcal{V D}$ a further notion of discrimination called q-discriminating was introduced and then it was proved that a group is squarelike if and only if it is q-discriminating.

Definition 7.1. (see [N]) Let $\mathcal{V}$ be a variety of groups and $G \in \mathcal{V} . G$ discriminates $\mathcal{V}$ provided to every finite set $w_{i}(\bar{x})$ of words in the varibales $X \cup X^{-1}$ with none of the equations $w_{i}(\bar{x})=1$ a law in $\mathcal{V}$ there is a tuple $\bar{g}$ of elements of $G$ such that simultaneously $w_{i}(\bar{g}) \neq 1$ in $G$ for all $i$. Further a group $G$ is varietally discriminating if it discriminates the variety it generates.

Theorem 7.1. $\mathcal{D} \subset \mathcal{S} \subset \mathcal{V D}$ and all inclusions are proper.

The proper inclusion $\mathcal{D} \subset \mathcal{S}$ was described already. The proper inclusion $\mathcal{D} \subset \mathcal{V D}$ can be proved independently of the middle. In order to prove the proper inclusion $\mathcal{S} \subset \mathcal{V} \mathcal{D}$ we introduce a quasivarietal version of varietal discrimination. 
Definition 2.1. Let $\mathcal{Q}$ be a quasivariety and let $G \in \mathcal{Q}$. Then $G$ qdiscrimina-tes $\mathcal{Q}$ provided that given finitely many quasilaws

$$
\forall \bar{x}\left(\left(\bigwedge_{i} u_{i}(\bar{x}=1) \rightarrow\left(w_{j}(\bar{x})=1\right)\right.\right.
$$

with the same antecedents and none of which holds in $\mathcal{Q}$ there exists a tuple $\bar{g}$ from $G$ such that simultaneously $u_{i}(\bar{g})=1$ and $w_{j}(\bar{g}) \neq 1$ for all $i, j . A$ group $G$ is q-discriminating if $G$ q-discriminates qvar $(G)$.

This almost, except for having the same antecedents, appears to be the translation of varietal discrimination in terms of quasivarieties. The condition of having the same antecedents is necessary for the next theorem. Without this restriction the only q-discriminating group would be the trivial group. The above notion of q-discrimination characterizes squarelike groups. We introduce one more idea.

Definition 7.2. A group $G$ is q-algebraically closed if and only if whenever a finite system

$$
\begin{gathered}
u_{i}\left(x_{1}, \ldots, x_{n}\right)=1 \\
w_{j}\left(x_{1}, \ldots, x_{n}\right) \neq 1
\end{gathered}
$$

of equations and inequations has a solution in some group $H \in q \operatorname{var}(G)$ it also has a solution in $G$.

We can now characterize squarelike groups in terms of q-discrimination.

Theorem 7.3. ([FGS 5]) Let $G$ be a group. The following are pairwise equivalent.

(1) $G$ is q-discriminating

(2) $G$ is q-algebraically closed

(3) $G$ is squarelike

$G$ is q-discriminating

\section{REFERENCES}

[GB] B.Baumslag, Lecture Notes on Nilpotent Groups, Amer. Math. Soc. Providence, RI., 1969.

[BFGS 1] G.Baumslag, B. Fine, A.M. Gaglione and D. Spellman, Reflections on Discriminating Groups, J. Group Theory, to appear.

[BFGS 2] G.Baumslag, B. Fine, A.M. Gaglione and D. Spellman, A Note on Nondiscrimination of Nilpotent Groups and Malcev Completions, Cont. Math. ,to appear.

[BMR 1] G. Baumslag, A.G. Myasnikov and V.N. Remeslennikov, Algebraic Geometry over Groups 1, J. of Algebra, 219, 1999, 16-79.

[BMR 2] G. Baumslag, A.G. Myasnikov and V.N. Remeslennikov, Discriminating and co-discriminating groups, J. of Group Theory, 3, 2000, 467-479.

[Be] O. Belegradek, Discriminating and square-like groups, J. Group Theory, 7, 2004, 521-532. 
[BS] J.L. Bell, A.B. Slomson, Models and Ultraproducts: An Introduction, NorthHolland, Amsterdam', 1972 (Second revised printing).

[D] V.H. Dyson Private Communication

[FGMS 1] B. Fine, A.M. Gaglione, A.G. Myasnikov and D. Spellman, Discriminating groups, J. of Group Theory, 4, 2001, 463-474.

[FGMS 2] B. Fine, A.M. Gaglione, A.G. Myasnikov and D. Spellman, $r$ Groups whose universal theory is axiomatizable by quasi-identities, J. of Group Theory, 5, 2002, 365-381.

[FGS 1] B. Fine, A.M. Gaglione and D. Spellman, Every abelian group universally equivalent to a discriminating group is elementarily equivalent to a discriminating group, Contemporary Math. 296 , $2002,129-137$.

[FGS 2] B. Fine, A.M. Gaglione and D. Spellman, , The axiomatic closure of the class of discriminating groups , Archiv. der Math., 83, 2004, 106-112.

[FGS 3] B. Fine, A.M. Gaglione and D. Spellman, Discriminating and Squarelike Groups I: Axiomatics, Contemporary Math., 360, 2004, 35-46.

[FGS 4] B. Fine, A.M. Gaglione and D. Spellman, , Discriminating and Squarelike Groups 2: Examples, Houston J. of Math., 31, 2005, 649-673.

[FGS 5] B. Fine, A.M. Gaglione and D. Spellman, Notions of Discrimination, Comm. in Alg. to appear.

[ Hi 1] G. Higman, Subgroups of finitely presented groups, Proc. Royal Soc. London Ser A, 264,1961), 455-475.

[Hi 2] G. Higman, Finitely Presented Infinite Simple Groups, Notes on Pure Math. 8, I.A.S., Austral. Nat. Univ., Canberra, 1974.

[HM] R. Hirshon and D. Meier, Groups with a quotient that the original group as a direct factor, Bull. Austral. Math. Soc., 45, 1992, 513-520.

[J] J.M. Tyrer Jones, Direct products and the Hopf property, J. Austral. Math. Soc., 17, 1974, 174-196.

[K] M.Kasabov, On Discriminating Solvable GroupsWeak Identities for Finitely Generated Groups, J. of Group Theory to appear.

[Ku] A.G. Kurosh, The Theory of Groups Vol. II, Chelsea, New York, 1956.

[MKS] W. Magnus, W., A. Karass, D. Solitar , Combinatorial Group Theory, Interscience, New York, 1966.

[MA] A. I. Mal'cev, On free soluble groups, Dokl. Akad. Nauk SSR, 130, 1960, 495498(English transl. Soviet Math. Dokl. (1960), 65-68.).

[MS] A.M. Myasnikov and P. Shumyatsky, Pn Discriminating Groups and c-Dimension, J. of Group Theory, 7 1, 2004, 135-142 to appear.

[Ne] B.H. Neumann, Some remarks on infinite groups, J. London Math. Soc. 12(1937), 120-127,

[N] H. Neumann, Varieties of Groups, Springer-Verlag, 1968.

[S] W. Szmielew, Elementary properties of Abelian groups, Fund. Math., 41, (1955), 203-271.

Benjamin Fine, Department of Mathematics, Fairfield University, Fairfield, Connecticut 06430, United States

Anthony M. Gaglione, Department of Mathematics, United States Naval Academy, Anapolis,Maryland 21402, United States

Alexei Myasnikov, Department of Mathematics, McGill University, Montreal, Quebec, Canada 
Dennis Spellman, Department of Mathematics, Temple University, PhiladelPhia, Pennsylvania 19132, United States 